\title{
Introduction to the Special Issue Epistemic Aspects of Many-Valued Logics
}

\author{
Timothy Childers $\cdot$ Ondrej Majer
}

Received: 13 January 2014/ Accepted: 13 January 2014/Published online: 31 January 2014

(C) Springer Science+Business Media Dordrecht 2014

The papers in this special issue are based on presentations delivered at the conference Epistemic Aspects of Many-valued Logics, held at the Institute of Philosophy of the Academy of Sciences of the Czech Republic, in Prague, 2010. All papers consequently revolve around the application of non-classical logical toolsmathematical fuzzy logic and/or probability theory-to epistemological issues.

Timothy Williamson employs a modal epistemic logic enriched with probabilities to generalize an argument against the KK-principle. He argues that we can know a proposition even if our evidential probability for that proposition is low. In fact he argues that the evidential probability of a known proposition can be (arbitrarily) close to 0 . The argument is first presented with a basic idealized model, which is then extended to much more complicated and realistic models. This then raises a problem for decision theory, since you can know that $p$, while your evidence tells you (strongly) that not $p$. Williamson argues that this problem should be understood as a failure of luminosity, i.e., that if you are in a state then you are in a position to know that you are in that state. Williamson also argues that a version of deductive closure of knowledge is defensible as long as we drop the KK-principle. Since knowledge states aren't luminous, standard arguments against closure don't work.

Colin Howson argues the subjective interpretation of the probability calculus should be understood in the framework of many-valued higher-order logics. Howson argues that the standard objections to second-order logic rely on a misguided conception of logic, and argues that we should instead concentrate on the model-theoretic notions of consistency and consequence. He then interprets the finitely additive probability calculus using these notions, linking fair bets with probabilities à la de Finetti. The restriction to finite additivity yields two logical properties: that a consistent probability assignment is extendable to a total assignment, and that an assignment is consistent if and only if all of its restrictions

T. Childers · O. Majer ( ( )

Prague, Czech Republic

e-mail: majer@flu.cas.cz 
to finite subsets are consistent. (These properties do not hold for a countably additive probability assignment.) Howson then examines-and dismisses-objections to finite additivity in light of these properties. He concludes that the probability calculus so interpreted is not subject to a problem of logical omniscience said to arise in the Bayesian context.

Vincenzo Marra attacks the problem of artificial precision for degree-theoretic approaches to vagueness. He uses the following observation to sharpen the nature of the problem: there is a one-to-one correspondence between the $[0,1]$ interval and sets of maximally consistent theories of infinite-valued Lukasiewicz propositional logic, where the theories are those propositions made true to degree one by an atomic proposition having truth degree $r$. This allows the transformation of the semantic question of what it means for a proposition to be true to degree $r$ to a syntactic question which is answerable solely by classically valued propositions. Thus, perhaps for the first time, the path is open to an elicitation (and hence epistemic application) of fuzzy values via a straightforward and intuitive connection of real numbers and propositions.

Nicholas Smith aims to show how subjective probabilities, degrees of belief and degrees of truth are related. He explicates degree of belief as a tendency to action. But this implies that there can't be two different kinds of degrees of belief, one fuzzy, and one probabilistic, as that would allow two different tendencies to action. Smith opts to interpret vagueness in terms of degrees of truth instead of epistemically. Degree of belief then has two components: the agent's epistemic state (which possible world is the actual one) and vagueness (the degree to which particular propositions hold in the actual world). The epistemic state is represented as a measure over the set of possible worlds, while degrees of truth are represented fuzzy-logically, and hence the agent's degree of belief in a proposition is the expected truth value of this proposition with respect to the probability measure.

We wish to thank the participants of the conference Epistemic Aspects of Manyvalued Logics for the inspirational atmosphere their active engagement created. Timothy Childers acknowledges the support of grant GAP401/10/1504, and Ondrej Majer of grant GA13-21076S, both of the Czech Science Foundation. We also thank the Institute of Philosophy for its support of the conference. 